\title{
Early effectiveness of type-2 severe asthma treatment with dupilumab in a real-life setting; a FeNO-driven choice that leads to winning management
}

\author{
Giovanna Elisiana Carpagnano, ${ }^{1 *}$ Giulia Scioscia, ${ }^{2 *}$ Enrico Buonamico, ${ }^{1}$ Donato Lacedonia, ${ }^{2}$ Fabrizio Diaferia, ${ }^{1}$ \\ Elena Capozza, ${ }^{1}$ Giorgia Lepore, ${ }^{2}$ Onofrio Resta, ${ }^{1}$ Maria Pia Foschino Barbaro ${ }^{2}$ \\ ${ }^{1}$ Institute of Respiratory Disease, Department of Basic Medical Sciences, Neuroscience and Sense Organs, University of Bari \\ “Aldo Moro", Bari \\ ${ }^{2}$ Department of Medical and Surgical Sciences, Institute of Respiratory Diseases, University of Foggia, Italy
}

\section{*These authors contributed equally to this work.}

Background: Dupilumab is a humanized monoclonal antibody targeting the IL4/IL13 signaling pathway, already used for atopic dermatitis and chronic rhinitis with nasal polyps, recently approved for severe type- 2 asthma. Its efficacy has been demonstrated in randomized control trials. The aim of our study is to evaluate possible early clinical improvement and type 2 biomarkers modifications in severe asthmatic patients treated with dupilumab in a real-life setting. Methods: We included 12 patients with severe, uncontrolled asthma and dupilumab was chosen if there was at least one evidence of blood eosinophils $>150$ cells/ml and/or FeNO>25 ppb during last year. Recent blood eosinophil count report, assessment through ACT, FeNO test and spirometry were performed at baseline and after 3 months of treatment. We calculated also the number of patients achieving a minimal, yet clinically relevant difference in $\mathrm{FEV}_{1}$ and ACT.

Results: After three months of treatment with dupilumab, ACT had a significant improvement (mean ACT pre 13.25 \pm 4.65 vs mean ACT post 19.17 \pm 4.45 ; $\mathrm{p}<0.01$ ), so as $\mathrm{FEV}_{1} \%$ (mean $\mathrm{FEV}_{1} \%$ pre $62.58 \pm 15.73$ vs mean $\mathrm{FEV}_{1} \%$ post 71.00 $\pm 13.11 ; \mathrm{p}<0.01$ ). FeNO had a significant reduction (median FeNO 32 pre, IQR 19-48.5 vs median FeNO19 post, IQR 16.5-26), differently from eosinophils blood count (median eosinophils pre 280, IQR 193.8-647.3 vs median

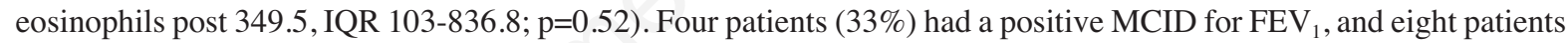
(67\%) had a positive MCID for ACT.

Conclusions: In RCTs performed during clinical development program dupilumab showed an early efficacy in increasing $\mathrm{FEV}_{1}$, reducing FeNO and improving asthma control. Our study demonstrates early improvement in asthmatic symptoms, lung function and FeNO in severe type- 2 asthma patients after only 3 months of dupilumab biologic therapy. The introduction of FeNO levels evaluation in the selection criteria for dupilumab, further helps the identification of eligible patients among type- 2 severe asthma patients and allows a complete outpatient assessment. Further real-life studies with a longer follow up time will be useful to confirm dupilumab efficacy and to promote its use in clinical practice.

Key words: Severe asthma; FeNO; dupilumab; real-life.

Correspondence: Enrico Buonamico, Institute of Respiratory Disease, Department of Basic Medical Sciences, Neurosciences and Sense Organs, University of Bari Aldo Moro, Piazza Giulio Cesare 11, 70125 Bari Italy.

E-mail: enricobuonamico@gmail.com

Contributions: GEC, GS, study conception and design; FD, EC, GL, data collection; EB, analysis and interpretation of results, draft manuscript preparation; DL, OS, MPFB, critical draft review. All the authors have read and approved the final version of the manuscript and agreed to be accountable for all aspects of the work.

Ethics approval and consent to participate: This study was carried out according to the principles of the Declaration of Helsinki, was approved by the local Ethics Committee of the "Riuniti" Hospital of Foggia (Institutional Review Board approval number 17/CE/2014). All recruited patients gave their written informed consent.

Conflict of interest: The authors declare no competing interests, and all authors confirm accuracy.

Availability of data and materials: The data supporting the findings of this study are available from the corresponding author on reasonable request. 


\section{Introduction}

Since the publication of 2014 ATS/ERS severe asthma guidelines [1], severe asthma is defined as requiring GINA step 4 or 5 of treatment to prevent it from becoming uncontrolled, or asthma not controlled despite these treatment steps.

Several biological treatments are already available for type- 2 endotype asthma, while none for type no 2.

The identification of type- 2 bronchial endotype includes the evaluation of eosinophils in peripheral blood cells count, a test with low specificity but easy to perform, and the research of eosinophils in induced sputum or into the bronchoalveolar lavage fluid, that gives more specific information as these markers express target organ inflammation, but more difficult to perform. Currently, operating the correct choice among biologics therapies for severe asthma is difficult because severe asthmatic patients are often atopic and this results in high count of blood eosinophils. Therefore, there is increasing interest in clinical practice in FeNO, a non-invasive and easily measurable type 2 biomarker that can help in the selection of the right biologic treatment for the right patient, guiding us towards personalized medicine in severe asthma.

Monoclonal antibody therapy plays an increasingly important role in the treatment of severe asthma and different targets have been identified [2]. Dupilumab is the latest monoclonal antibody approved by the Italian Drug Agency (Agenzia Italiana del Farmaco - AIFA) for severe asthma treatment, while it was already available for atopic dermatitis treatment. Its mechanism of action is centered onto IL-4 and IL-13 signaling pathways: Th2 cells, mast cells, eosinophils, basophiles, CD8 T cells and Natural Killer cells stimulate the secretion of IL-4 and IL-13. These cytokines drive Ig class switching from $\operatorname{IgM}$ to $\operatorname{IgE}$ in B lymphocytes and plasma cells; IL-4 and IL-13 enhance airway muscle contractility and induce eosinophils recruitment in airways mucosa. IL-13 stimulates mucus production and epithelial cell expression of inducible nitric oxide synthase; it also promotes significant airway remodeling in asthma by enhancing goblet cell hyperplasia, transformation of bronchial fibroblasts into myofibroblast, deposition of collagen, and proliferation of airways smooth muscle cells.

Dupilumab is a fully human monoclonal antibody. It is directed against the alpha subunit of the IL-4 receptor, thereby blocking both IL-4 and IL-13 signaling, hence type 2 inflammation. In addition to randomized control trials (RCTs), Xiong et al. also assessed the safety of dupilumab in a meta-analysis involving 3,369 patients, showing that the biological drug is relatively well tolerated and could significantly improve $\mathrm{FEV}_{1}$, symptoms, asthma control, and quality of life by reducing exacerbations and risks related to uncontrolled asthma [3]. Dupilumab, already used for the treatment of moderate to severe atopic dermatitis and for the treatment of chronic rhinosinusitis with nasal polyposis (CRSwNP) [4,5], recently obtained a new therapeutic indication that other biological treatments do not have yet: it is indicated in severe asthma with type 2 inflammation, in adults and adolescents aged 12 years and over, characterized by an increased number of eosinophils in blood and/or FeNO, not adequately controlled with high-dose ICS and another medicinal product for maintenance treatment.

Our study aims to evaluate in a real-life setting early clinical improvement and modifications of Type- 2 inflammation markers after 3 months of add-on treatment with dupilumab in severe asthmatic patients.

\section{Methods}

This is a retrospective dual-center real-life study. Data were collected between June and December 2020 at the severe asthma dedicated outpatients' clinics of respiratory units of the University hospitals of Bari and Foggia.

This study was carried out according to the principles of the Declaration of Helsinki, was approved by the local Ethics Committee of the "Riuniti" Hospital of Foggia (Institutional Review Board approval number 17/CE/2014), and all recruited patients gave their written informed consent.

We included twelve patients to whom we presented with the possibility of a step-up to biologic treatment if they had a personal history of severe uncontrolled asthma, following 2014 ATS/ERS guidelines [1], despite treatment with step IV GINA therapy. Dupilumab was administered, in agreement with the patient, if there was at least one evidence of blood eosinophils more than 150 cells/ml and/or FeNO more than 25 ppb in the last year. Dupilumab was chosen among different monoclonal antibodies options following the evidence of high FeNO levels, a clear marker of IL4/IL-13 pathway upregulation. Dupilumab was administered subcutaneously every two weeks with a loading dose of $400 \mathrm{mg}$ and a maintenance dose of $200 \mathrm{mg}$ in our outpatients' clinics, following the administration protocol. We collected personal history of each patient, focusing on symptoms evaluation through ACT, and blood analysis reports with peripheral blood eosinophils count (samples collected less than 2 weeks before the examination), then all patients underwent FeNO test and spirometry. This routine was performed both at baseline and after three months of treatment. Moreover, at baseline we evaluated serum total IgE. In addition to the evaluation of clinical improvement and type-2 biomarkers response to dupilumab, we calculated the percentage of patients that had a minimal clinically important improvement in lung function (improvement of $15 \%$ from baseline) and symptoms control (improvement of 3 points of ACT from baseline) [6].

All the parameters of this study were tested for normality with Kolmogorov-Smirnov test. For non-parametric values, comparison was made with Wilcoxon matched-pairs signed rank test, while for parametric values comparison was made with paired $t$-test.

\section{Results}

Six patients of the total population were from outpatient's clinic in Bari and six from Foggia. Patients' general parameters are reported in Table 1. Our population median age was 64 years old (IQR 44.25-71), female patients were 5 (42\%). All our patients were affected by late-onset asthma. Four patients (33\%) had obesity, while 8 patients $(67 \%)$ had allergic comorbidities. Mean total serum IgE at first visit was $182,67( \pm 106.76)$, median blood eosinophils at admission was 280 (193.8-647.3). At the time of the enrollment, all patients were on high dose ICS/LABA therapy, and 10 patients out of them $(83 \%)$ were receiving LAMA add-on therapy. Ten patients $(83 \%)$ were already receiving chronic OCS treatment, with mean daily dosage $5.8 \mathrm{mg}( \pm 2.59)$, two $(17 \%)$ patients were on anti-IgE therapy omalizumab, two (17\%) patients were on anti-IL-5R therapy benralizumab and one $(8 \%)$ patient was on anti-IL5 therapy mepolizumab and were all switched to dupilumab due to a lack of response to the previous biologic treatment. In particular, patients that were under treatment with omalizumab had a longer time on treatment (respectively 3 and 4 years) and experienced a reduction in drug efficacy over years, while patients under anti-eosinophil targeted therapy (benralizumab and mepolizumab) 
were switched to dupilumab because of evidence of insufficient amelioration of perceived asthmatic symptoms and asthmatic exacerbations despite evidence of blood eosinophil count reduction.

Comparison between parameters collected in the first and second visits are shown in Table 2. After three months of treatment with dupilumab, ACT had a significant improvement (mean ACT pre 13.25 \pm 4.65 vs mean ACT post $19.17 \pm 4.45$; $\mathrm{p}<0.01$ ) (Figure 1b), so as $\mathrm{FEV}_{1} \%$ (mean $\mathrm{FEV}_{1} \%$ pre $62.58 \pm 15.73$ vs mean $\mathrm{FEV}_{1} \%$ post $71.00 \pm 13.11 ; \mathrm{p}<0.01$ ) (Figure 1a). FeNO after three months showed a significant reduction (median FeNO pre 32, IQR 19-48.5 vs median FeNO post 19, IQR 16.5-26) (Figure 1c), while we observed a relative increase in eosinophils blood count, despite it did not reach statistical significance and no clinical impact (median eosinophils pre 280, IQR 193.8-647.3 vs median eosinophils post 349.5, IQR 103-836.8; p=0.52) (Figure 1d). Four patients $(33 \%)$ achieved a positive MCID for $\mathrm{FEV}_{1}$, and eight patients (67\%) had a positive MCID for ACT.

\section{Discussion}

Although dupilumab has so far demonstrated its efficacy in the treatment of atopic dermatitis, both in randomized control trials and in real-life studies $[7,8]$, there is a lack of published evidence of dupilumab efficacy in severe asthma treatment in real-life. With our findings, we show that dupilumab, in severe uncontrolled asth-

Table 1. General parameters. Non-parametric variables are expressed in median (IQR) and parametric variables are expressed in mean $( \pm S D)$, proportions are expressed in absolute value (percentage).

\begin{tabular}{lc}
\hline Number of patients & 12 \\
Age (years) & $64(44.25-71)$ \\
\hline Female & $5(42 \%)$ \\
Obesity & $4(33 \%)$ \\
\hline Allergic comorbidities & $8(67 \%)$ \\
Total serum IgE at first examination & $182.67( \pm 106.76)$ \\
\hline Blood eosinophils at first examination & $280(193.8-647.3)$ \\
Patients on high dose ICS/LABA & $12(100 \%)$ \\
Patients on LAMA & $10(83 \%)$ \\
Patients on chronic OCS & $10(83 \%)$ \\
\hline Patients on omalizumab & $2(17 \%)$ \\
Patients on mepolizumab & $1(8 \%)$ \\
\hline Patients on benralizumab & $2(17 \%)$
\end{tabular}

OCS, oral corticosteroids.

Table 2. Comparison between first (T0- baseline) and second visit after 3 months (T1). Non parametric variables are expressed in median (IQR), parametric variables are expressed in mean $( \pm \mathrm{SD})$.

\begin{tabular}{lccc} 
& T0 & T1 & p \\
ACT & $13.25( \pm 4.65)$ & $19.17( \pm 4.45)$ & $<0.01$ \\
FEV $_{1} \%$ & $62.58( \pm 15.73)$ & $71( \pm 13.11)$ & $<0.01$ \\
FeNO & $32(19-48.5)$ & $19(16.5-26)$ & $<0.05$ \\
Blood eosinophils & $280(193.8-647.3)$ & $349.5(103-836.8)$ & 0.52 \\
\hline
\end{tabular}

matic patients of type 2 endotype, has clinical efficacy as soon as after 3 months of treatment, improving symptoms, lung function and $\mathrm{FeNO}$.

In the RCTs performed during the clinical development program, dupilumab showed an early efficacy in pre-bronchodilator $\mathrm{FEV}_{1}$ increase, evident from the second week of treatment $[9,10]$ and furtherly remarked when patients were stratified by blood eosinophil count, with a higher efficacy in patients with $>300$ cells $/ \mu 1$ [11]. The results of our study confirm these findings, showing a significant increase in pre-bronchodilator $\mathrm{FEV}_{1}$ in our patients after 3 months, which is associated to symptoms amelioration, underlined by ACT significant increase, and reduction in T2 biomarker FeNO.

We found only two real-life study describing dupilumab effect in severe asthma. Dupin et al. in a multi-centre retrospective cohort study show dupilumab efficacy in 64 severe asthma patients for $\mathrm{FEV}_{1}$ improvement, ACT improvement, daily prednisone dose reduction and annual exacerbation rate reduction after one year of treatment [12]. This study, as compared to ours, has a longer timespan observation, and takes into account also corticosteroid usage and exacerbation rate. Conversely, our study describes a smaller cohort with a shorter follow up time, but we added questionnaires to evaluate the quality of life linked to asthma symptoms burden, that further completes a rounded assessment. Adding new patients to the present cohort and prolonging the follow up time could let us observe a reduction in exacerbations and a decrease in systemic corticosteroid dosage.

Mummler et al. analysed 38 patients affected by severe asthma in biologic therapy with anti-IgE, anti-IL5/IL5R at the time of the enrollment, switched to dupilumab, and showed 6 months efficacy in ACT improvement, $\mathrm{FEV}_{1}$ improvement, FeNO reduction, exacerbation reduction and decreasing in patients requiring OCS [13]. Another interesting finding of this study is the higher rate of responders in patients with baseline FeNO $>25 \mathrm{ppb}$. In our study 5 patients $(42 \%)$ were switched to dupilumab from a previous biologic therapy for severe asthma, and the numerosity of the cohort is insufficient to draw conclusions about this specific subgroup.
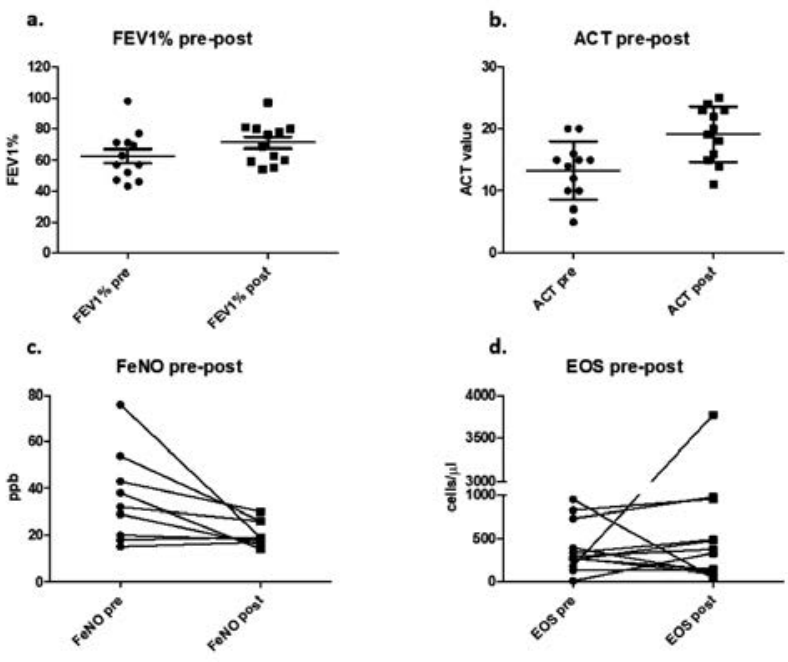

Figure 1. a) $\mathrm{FEV}_{1} \%$ baseline $v s \mathrm{FEV}_{1} \%$ after 3 months. b) ACT baseline $v s$ ACT after 3 months. c) FeNO baseline $v s$ FeNO after 3 months. d) Blood eosinophils baseline $v s$ blood eosinophils after 3 months. 
The implementation of $\mathrm{FEV}_{1}$ and ACT MCID evaluation in our patients may be helpful in order to assess treatment impact in each patient, an approach that could lead us to a complete assessment of patient's health, identifying the number of patients that experience a clinically significant improvement in lung function and symptoms control. It is interesting to underline that the number of patients with a positive MCID for ACT is higher than the percentage of patients with a positive MCID for $\mathrm{FEV}_{1}$. This may indicate that, like in other biologic drugs for severe asthma, dupilumab main early effect is asthma stabilization, also suggested by FeNO reduction. Our patients showed an elevation in peripheral blood eosinophils, evidence reported both by RCTs [9-11] and by Dupin et al. [12] that surely deserves attention, in order to deepen our knowledge on the homeostatic pathways in type- 2 asthma. The unique mechanism of action of dupilumab gives us the opportunity to add another biomarker to those usually considered in the choice of the appropriate biologic drug in severe asthma, FeNO test. Despite the well-known role of FeNO in the diagnosis, phenotyping and follow up of type- 2 asthma, suggested by the latest severe asthma GINA guidelines [14] and lately in a position paper by Italian Respiratory Society and Italian Society of Allergy, Asthma and Clinical Immunology [15], by far the criteria for the prescription of biologic drugs in severe asthma included $\operatorname{IgE}$ dosage for anti-IgE drug and peripheral blood eosinophil count for anti IL5 and anti IL5R drugs. With the introduction of dupilumab in type 2 severe asthma, FeNO becomes a key biomarker, following the evidence of IL13 role in iNOS induction in bronchial epithelial cells that determines a rise in exhaled nitric oxide $[16,17]$. The everwider choice among biologic drugs that target different molecules that are involved in the same type of inflammation in asthma raises new questions for respiratory physicians on how to make the right decision between the available options. The introduction of FeNO levels evaluation in the selection criteria for dupilumab, at the moment the only biologic drug with this feature, further helps the identification of eligible patients among all type- 2 severe asthma patients, placing another step towards precision medicine in asthma. Another matter of importance about FeNO test for respiratory physicians is therefore linked to the possibility of a complete and fast outpatient clinic-centered patients' assessment, that may facilitate diagnostic-therapeutic routes in severe asthma management.

A limitation of this study is the small number of subjects enrolled, being dupilumab available for asthma in our country from relatively short time.

\section{Conclusions}

Our dual center real-life study demonstrates early improvement in asthmatic symptoms, lung function and FeNO in severe type- 2 asthma patients after 3 months of dupilumab biologic therapy, a result that is in line with RCTs and with the few other reallife studies published so far. In a future study, we will focus our attention to evaluate long-time real-life efficacy of dupilumab, thanks to a longer follow up time.

\footnotetext{
Abbreviations

FeNO: fractionexhaled-nitrous-oxyde; $\mathrm{FEV}_{1}$ : forced exhaled volume $1^{\text {st }}$ second; ICS: inhaled corticosteroids; OCS: oral corticosteroids; ACT: asthma control test;
}

ATS: American Thoracic Society; ERS: European Respiratory Society; GINA: global initiative for asthma.

\section{References}

1. Chung KF, Wenzel SE, Brozek JL, Bush A, Castro M, Sterk PJ, et al. International ERS/ATS guidelines on definition, evaluation and treatment of severe asthma. Eur Respir J 2014;43:34373.

2. Busse WW. Biological treatments for severe asthma: A major advance in asthma care. Allergol Int 2019;68:158-66.

3. Xiong XF, Zhu M, Wu HX, Fan LL, Cheng DY. Efficacy and safety of dupilumab for the treatment of uncontrolled asthma: A meta-analysis of randomized clinical trials. Respir Res 2019;20:1-11.

4. Bachert C, Han JK, Desrosiers M, Hellings PW, Amin Nikhil, Lee SE, et al. Efficacy and safety of dupilumab in patients with severe chronic rhinosinusitis with nasal polyps (LIBERTY NP SINUS-24 and LIBERTY NP SINUS-52): results from two multicentre, randomised, double-blind, placebo-controlled, parallel-group phase 3 trials. Lancet.2019;394:1638-50.

5. Hoy SM. Dupilumab: A review in chronic rhinosinusitis with nasal polyps. Drugs 2020;80:711-7.

6. Bonini M, Di Paolo M, Bagnasco D, Baiardini I, Braido F, Caminati M, et al. Minimal clinically important difference for asthma endpoints: An expert consensus report. Eur Respir Rev 2020;29:1-14.

7. Halling AS, Loft N, Silverberg JI, Guttman-Yassky E, Thyssen JP. Real-world evidence of dupilumab efficacy and risk of adverse events: A systematic review and meta-analysis. J Am Acad Dermatol 2021;84:139-47.

8. Deleuran M, Thaçi D, Beck LA, de Bruin-Weller M, Blauvelt A, Forman S, et al. Dupilumab shows long-term safety and efficacy in patients with moderate to severe atopic dermatitis enrolled in a phase 3 open-label extension study. J Am Acad Dermatol 2020;82:377-88.

9. Castro M, Corren J, Pavord ID, Maspero J, Wenzel S, Rabe $\mathrm{KF}$, et al. Dupilumab efficacy and safety in moderate-to-severe uncontrolled asthma. N Engl J Med 2018;378:2486-96.

10. Rabe KF, Nair P, Brusselle G, Maspero JF, Castro M, Sher L, et al. Efficacy and safety of dupilumab in glucocorticoiddependent severe asthma. N Engl J Med 2018;378:2475-85.

11. Wenzel S, Castro M, Corren J, Maspero J Wang L, Zhang B, et al. Dupilumab efficacy and safety in adults with uncontrolled persistent asthma despite use of medium-to-high-dose inhaled corticosteroids plus a long-acting $\beta 2$ agonist: a randomised double-blind placebo-controlled pivotal phase $2 \mathrm{~b}$ dose-ranging trial. Lancet 2016;388:31-44.

12. Dupin C, Belhadi D, Guilleminault L, Gamez AS, Berger P, De Blay $\mathrm{F}$, et al. Effectiveness and safety of dupilumab for the treatment of severe asthma in a real-life French multi-centre adult cohort. Clin Exp Allergy 2020;50:789-8.

13. Mümmler C, Munker D, Barnikel M, Veit T, Kayser MZ, Welte $\mathrm{T}$, et al. Dupilumab improves asthma control and lung function in patients with insufficient outcome during previous antibody therapy. J Allergy Clin Immunol Pract 2021;9:1177.85.e4.

14. Global Initiative for Asthma [Internet]. GINA Difficult-to-treat \& severe asthma in adolescent and adult patients - Diagnosis and management. 2019. Available from: https://ginasthma.org/wp-content/uploads/2019/04/GINASevere-asthma-Pocket-Guide-v2.0-wms-1.pdf 
15. Heffler E, Carpagnano GE, Favero E, Guida G, Maniscalco M, Motta A, et al. Fractional exhaled nitric oxide (FENO) in the management of asthma: a position paper of the Italian Respiratory Society (SIP/IRS) and Italian Society of Allergy, Asthma and Clinical Immunology (SIAAIC). Multidiscip Respir Med 2020;15:36.

16. Chibana K, Trudeau JB, Mustovitch AT, Hu H, Zhao J, Balzar
S, et al. IL-13 induced increases in nitrite levels are primarily driven by increases in inducible nitric oxide synthase as compared with effects on arginases in human primary bronchial epithelial cells. Clin Exp Allergy 2008;38:936-46.

17. Suresh V, Mih JD, George SC. Measurement of IL-13-induced iNOS-derived gas phase nitric oxide in human bronchial epithelial cells. Am J Respir Cell Mol Biol 2007;37:97-104.

Received for publication: 25 July 2021. Accepted for publication: 22 November 2021.

This work is licensed under a Creative Commons Attribution-NonCommercial 4.0 International License (CC BY-NC 4.0).

CCopyright: the Author(s), 2022

Licensee PAGEPress, Italy

Multidisciplinary Respiratory Medicine 2022; 17:797

doi:10.4081/mrm.2022.797 